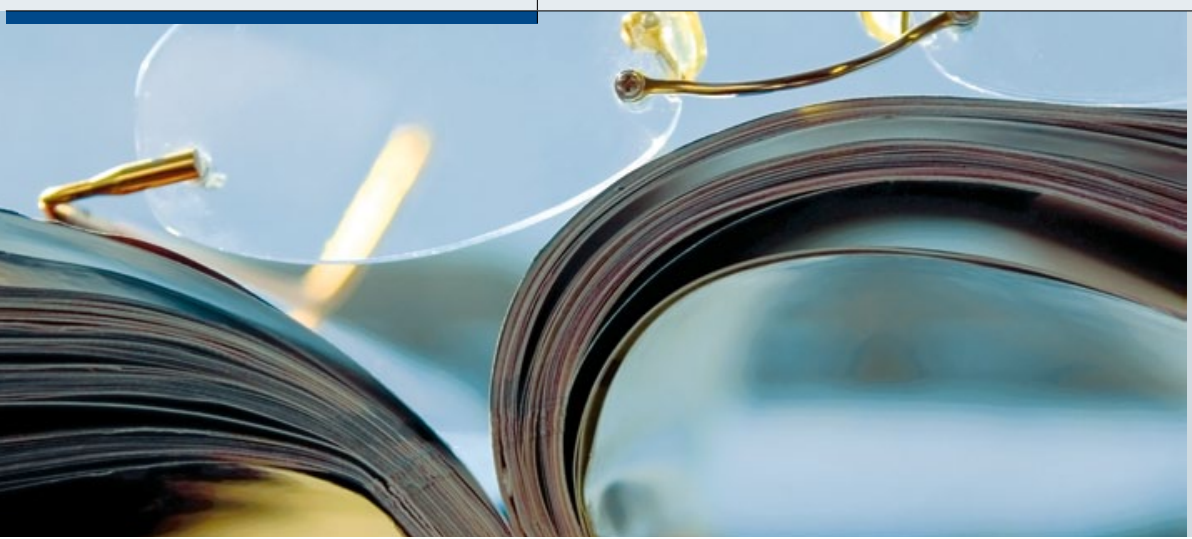

In der Rubrik "Literatur kompakt" werden wichtige Arbeiten aus der internationalen Fachliteratur referiert und auf den Punkt gebracht.

\section{Schleudertrauma: intensive Physiotherapie bietet keine Vorteile}

Nach einem Schleudertrauma werden anstelle einer Ruhigstellung mittels Halskrause heutzutage eine aktive Physiotherapie und intensive Edukation empfohlen. Frühe und intensive Physiotherapie soll das Chronifizierungsrisiko reduzieren, für die Edukation wurde eine Patientenbroschüre entwickelt. Lamb et al. haben nun erstmals in einer klinischen Studie untersucht, ob diese Maßnahmen wirksam und kosteneffektiv sind.

$\mathrm{D}$ ie Studie wurde in zwei Stufen durchgeführt. In Stufe 1 wurden Kliniken ausgewählt und entweder „usual care" oder ,,active management" zugeordnet. Patienten, die sich bis zu sechs Wochen nach einem Schleudertrauma Grad I-III (Quebec-Klassifikation) vorstellten, wurde eine Studienteilnahme angeboten, wenn sie drei Wochen später noch unter Beschwerden leiden sollten. In der Gruppe des ,active management" wurde die Übergabe der Patientenbroschüre mit der Aufforderung zur Aktivität verknüpft. Bei „usual care“ erfolgte die in der Klinik jeweils übliche Standardbehandlung.

In Stufe 2 erhielten die Patienten randomisiert bis zu sechs physiotherapeutische Behandlungen in acht Wochen oder eine einmalige Edukation mit standardisierter Untersuchung und Verhaltensempfehlungen (Advice). Der Schweregrad des Schleudertraumas sowie die resultierende Beeinträchtigung wurden dokumentiert. Nachbefragungen fanden nach vier, acht und zwölf Monaten statt. Erfasst wurden Beeinträchtigung (Neck Disability Index; NDI), Lebensqualität (SF-12, EQ-5D), Arbeitsunfähigkeitstage sowie Ressourcenverbrauch im Gesundheitssystem.

3.851 Patienten (mittleres Alter 37 Jahre) wurden in die Studie einge- schlossen. 949 Patienten konnten in Stufe 2 aufgenommen und 599 randomisiert werden (300 zur intensiven Physiotherapie, $299 \mathrm{zu}$ Advice). Weder nach vier, noch nach acht oder zwölf Monaten unterschieden sich die Gruppen der Stufe 1 im NDI, SF-12 oder bei den Tagen mit Ausfall am Arbeitsplatz. Nach vier, acht und zwölf Monaten fanden sich geringe Unterschiede bei der Beeinträchtigung, der NDI war nach vier Monaten signifikant geringer, nach acht und zwölf Monaten nicht mehr. Der SF-12 unterschied sich zu keinem Zeitpunkt, die Selbsteinschätzung war nur nach vier Monaten in der Physiotherapiegruppe signifikant besser. Es bestand ein geringer Vorteil der Physiotherapiegruppe hinsichtlich der Ausfalltage am Arbeitsplatz, jedoch waren die Therapiekosten in dieser Gruppe höher. Ärztliche Konsultationen, Einnahme von Analgetika und Inanspruchnahme von Gesundheitsressourcen waren nicht unterschiedlich.

Fazit: Zusätzliche Therapieintensivierungen mit Schulung und intensiver physiotherapeutischer Behandlung zeigen bei Patienten mit Schleudertrauma allenfalls einen kurzfristigen Effekt, haben aber keinen Einfluss auf das Therapieergebnis nach acht oder zwölf Mona- ten. Diese Maßnahmen sind nicht kosteneffizient.

Dr. Beate Schumacher

Lamb SE, Gates S, Williams MA et al. Managing Injuries of the Neck Trial (MINT) Study Team. Emergency department treatments and physiotherapy for acute whiplash: a pragmatic, two-step, randomised controlled trial. Lancet 2013; 381: 546-56

Kommentar: Das Ergebnis enttäuscht alle, die sich intensiv um die Aktivierung und Behandlung von Patienten mit einem Schleudertrauma bemühen. Ärzte wollen etwas gegen die Beschwerden ihrer Patienten unternehmen und Patienten erwarten das auch. Eine Verordnung von sechs Physiotherapie-Einheiten wird dankbar angenommen, trägt jedoch nicht zur Verbesserung des Verlaufs bei. Wahrscheinlich ist der Einwand, noch mehr Therapie wäre vielleicht nötig gewesen, nicht unberechtigt. Aber wir kennen alle Patienten, die über lange Zeit eine - teilweise auch hochfrequente - Physiotherapie erhalten haben, ohne dass ihre Beschwerden zurückgegangen wären. Die Durchführung der Untersuchung als pragmatische klinische Studie mit zahlreichen Patienten bildet den Versorgungsalltag viel näher ab als eine kontrollierte klinische Studie mit nur kleiner Fallzahl. Wenn wir uns dem Rat der Autoren anschließen, erhalten Patienten mit Schleudertrauma eine sorgfältige Aufklärung über das Krankheitsbild und Empfehlungen, um zum Beispiel einem Bewegungsvermeidungsverhalten entgegenzuwirken, aber keine Folgerezepte für zahllose physiotherapeutische Behandlungen.

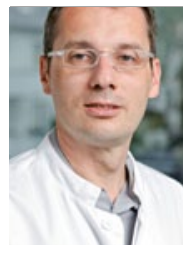

PD Dr. med. Charly Gaul Chefarzt der Migräne- und Kopfschmerzklinik Königstein E-Mail: c.gaul@migraeneklinik.de 